\title{
Habitual reading biases in the allocation of study time
}

\author{
Robert Ariel • Ibrahim S. Al-Harthy • \\ Christopher A. Was • John Dunlosky
}

Published online: 7 July 2011

(C) Psychonomic Society, Inc. 2011

\begin{abstract}
Item order can bias learners' study decisions and undermine the use of more effective allocation strategies, such as allocating study time to items in one's region of proximal learning. In two experiments, we evaluated whether the influence of item order on study decisions reflects habitual responding based on a reading bias. We manipulated the order in which relatively easy, moderately difficult, and difficult items were presented from left to right on a computer screen and examined selection preference as a function of item order and item difficulty. Experiment 1a was conducted with native Arabic readers and in Arabic, and Experiment $1 \mathrm{~b}$ was conducted with native English readers and in English. Students from both cultures prioritized items for study in the reading order of their native language: Arabic readers selected items for study in a right-to-left fashion, whereas English readers largely selected items from left to right. In Experiment 2, native English readers completed the same task as participants in Experiment 1b, but for some participants, lines of text were rotated upside down to encourage them to read from right to left. Participants who read upsidedown text were more likely to first select items on the right side of an array than were participants who studied right-side-up text. These results indicate that reading habits can bias learners' study decisions and can undermine agenda-based regulation.
\end{abstract}

Keywords Self-regulated study $\cdot$ Metacognition

R. Ariel $(\bowtie) \cdot$ I. S. Al-Harthy $\cdot$ C. A. Was · J. Dunlosky

Psychology Department, Kent State University,

Kent, OH 44242, USA

e-mail: rariel@kent.edu
Research on the allocation of study time has demonstrated that the difficulty of materials influences how long students study them and which are selected for study (for reviews, see Dunlosky \& Ariel, 2011; Son \& Kornell, 2009). For instance, when students have limited time for study, they often first select the easiest unlearned materials to study and then transition to progressively more difficult ones (Metcalfe, 2009). According to the agenda-based regulation framework (Ariel, Dunlosky, \& Bailey, 2009), students are developing an agenda-or simple plan-to help them maximize performance. This particular agenda has been called using a region of proximal learning (RPL; Metcalfe, 2002), and using it can improve learning (Kornell \& Metcalfe, 2006). Recently, however, Dunlosky and Ariel (in press) reported that how materials were presented influenced students' item selections and undermined their use of an RPL agenda.

In particular, Dunlosky and Ariel (in press) examined the influence of the presentation order of items on studytime allocation for relatively easy, moderately difficult, and difficult English-Spanish translations. On each trial, three English words were presented side by side in a row, and participants chose translations to study by clicking a button below each English word. Item difficulty (i.e., easy, moderate, or difficult) was presented above each English word. Items were ordered from left to right either from the easiest to the most difficult item or from the most difficult to the easiest item. Learners often chose to study the easier items first, which demonstrated the use of an RPL agenda (Metcalfe, 2009), but their decisions were also influenced by presentation order. That is, learners often first chose to study the item in the left position of the array, followed by the middle item, and then finally the right item. 
Why are learners' allocation decisions influenced by the order of items in an array? Dunlosky and Ariel (in press) speculated that this influence is due to the directional reading habits of one's language, which biases one to select items for study in the order that they are encountered during reading. Though these behavioral patterns indicate an itemorder bias for study-time allocation, the hypothesis that reading habits produce this bias was not evaluated. Our goal was to evaluate this hypothesis using both a crosscultural analysis (Experiment 1a and 1b) and an experimental design (Experiment 2).

Reading habits influence people's preferences, decision making, perceptual-motor performance, attentional momentum, and how they represent numbers and time (e.g., Chokron \& De Agostini, 1995, 2000; Eviator, 1995; Shaki, Fischer, \& Petrusic, 2009; Spalek \& Hammad, 2005; Van der Henst \& Schaeken, 2005). In most of the studies listed here, the effects of reading habits on cognition were examined using cross-cultural comparisons of readers with a left-to-right (e.g., English) orthographic system to readers with a right-to-left (e.g., Arabic) orthographic system. A typical finding from these studies was that the structure of language biases task performance in a manner that is consistent with the scanning direction of one's native language (for a review, see Ouellet, Santiago, Israeli, \& Gabay, 2010). Given that directional reading habits influence cognition in a variety of domains, we suspect that reading habits may also bias study choices, and hence may be responsible for the item order biases observed in Dunlosky and Ariel (in press).

In the present experiments, we evaluated the degree to which reading habits bias study choices by manipulating the presentation order of items relative to how difficult an item was to learn. On each trial, three items were presented (one relatively easy, one moderately difficult, and one difficult to learn), and participants selected items for study. The order in which items were presented from left to right was varied across trials. On some trials, reading order was consistent with using an RPL agenda (with the easier items in the most prominent reading position), and on other trials, reading order was inconsistent with the use of an RPL agenda. The latter trials would allow one to evaluate the degree to which participants' study choices were based on using an RPL agenda or were biased by reading habits.

Experiment 1a was presented entirely in Arabic to native Arabic readers, and Experiment $1 \mathrm{~b}$ was presented in English to native English readers. Most relevant here were predictions about the influence of reading habits. Given that the Arabic and English languages are read in different directions (right to left and left to right, respectively), if directional reading habits bias study decisions, native Arabic readers will first select items positioned on the right side of an array, followed by items in the middle position, and so on. By contrast, native English readers should prefer selecting items in the reverse order. Alternatively, if reading habits did not bias learners' study choices, one would expect the participants to consistently allocate their study to items in their RPL first, regardless of item order. That is, learners from both cultures should first select the easiest unlearned item for study and then move on to progressively more difficult items (Metcalfe, 2009).

In Experiment 2, we evaluated the effects of directional reading habits on study decisions using an experimental manipulation that we expected would induce a right-to-left selection bias in some native English speakers. Participants again selected English-Spanish translations for study from a three-item array, but for some participants, each line of text was rotated $180^{\circ}$. Thus, if one's reading habits influence study decisions, participants should be more likely to choose items on the right side of an array first when text is presented rotated upside down as compared to when text is presented normally.

\section{Experiment 1a}

\section{Method}

Participants A group of 27 undergraduate students from the college of education at Sultan Qaboos University in Muscat, Oman, volunteered to participate. All participants were native Arabic speakers enrolled in an educational psychology course or a measurement course. Participants learned about the experiment from their instructor and were not compensated for participating.

Materials and procedures A total of 54 Arabic-English translations, consisting of 18 easy pairs (e.g., كتاب -"book”), 18 moderately difficult pairs (e.g., متعة -"pleasure"), and 18 difficult pairs (e.g., تسلية -"entertainment"), were used. Translations were taken from a pool of 147 translations, and then, based on outcomes from a pilot experiment, we chose 18 for each difficulty level. The task was presented in Arabic. Participants were instructed that they would be learning Arabic-English vocabulary pairs and that their goal was to learn the English translations. They were instructed that some words would be easier to learn, some would be moderately difficult, and others would be relatively difficult to learn. They were told that item difficulty (easy, moderate, or difficult) would be presented above each Arabic word during the study phase and that they could allocate study time however they wished.

On each study trial, three Arabic words (easy, moderately difficult, and difficult) were presented in a row on a computer screen. On each trial, items could be ordered from left to right in one of six ways: easy to moderate to difficult (i.e., EMD), EDM, MED, MDE, DEM, or DME. Participants received 
each order three times across trials. Trials were presented in blocks of six that included every order, and order was randomized within each block. On each study trial, the participants had $15 \mathrm{~s}$ to study and choose an English translation by clicking a button positioned below the corresponding Arabic word. When selected, the translation appeared on the screen and remained until time expired for the trial or another item was selected. After the final study trial, participants were tested on all of the Arabic-English translations: When each Arabic word was presented, participants attempted to type the English translation.

\section{Results and discussion}

Only effects with $p<.05$ are reported. We examined the order of item selection for each participant, conditionalized on presentation order. ${ }^{1}$ The proportions of times each participant selected items first, second, or third as a function of their position in the array were computed. We present an analysis only of first selections, because subsequent selections were consistent with a reading bias: The middle item was most often chosen second, and the leftmost item was most often chosen third.

Means across participants' values for their first choice are presented in the left panel of Fig. 1. Order condition is presented on the $x$-axis, and the bars refer to the positions from which items were selected from the array (left, middle, or right). The figure is easily interpreted if you consider the EMD condition on the far left. Arabic learners most often first chose the items on the right (darkest bar), which were the most difficult items (rightmost bar is above D, in EMD). Scanning the entire left panel, it is evident that the highest value for each condition (EMD, EDM, etc.) is the rightmost bar, indicating a right-to-left bias in first-choice selections. This observation was confirmed by a 2 (position selected: left or right) $\times 6$ (presentation order condition) ANOVA. The middle position in this ANOVA was not included, because once values are known for two levels (e.g., the left and right positions), the third value is fixed. The ANOVA revealed only a significant effect for position selected, $F(1,151)=31.98$, $M S E=7.19, \eta_{\mathrm{p}}{ }^{2}=.18$, indicating that participants more often selected items on the right with their first study choice.

Although it was not relevant to our focal aim, we also examined the proportions of items recalled as a function of presentation order condition. Recall performance for each experiment is presented in the Appendix. Given that effects of order were not present (see the note to Table 1), one may question whether using an RPL agenda mattered. As

\footnotetext{
${ }^{1}$ Self-paced study times were not relevant to our focal aim, and hence are not reported, but they are available from the first author.
}

discussed by Dunlosky and Ariel (in press), the present method may not be sensitive to the benefits of using an RPL agenda, which other research has shown to be effective (Atkinson, 1972; Kornell \& Metcalfe, 2006). Even so, differences in selection order may not matter as long as participants have sufficient time to study all of the items, and in the present experiments, participants typically selected all three items per trial. In the General Discussion, we discuss a context in which reading habits disrupt the use of an agenda that is unquestionably effective.

\section{Experiment 1b}

In Experiment 1a, native Arabic readers selected items for study in a right-to-left fashion, which confirms the hypothesis that item selection is biased by reading habits. This result is consistent with the results of Dunlosky and Ariel (in press), who found that English readers selected items in the opposite fashion. In contrast to Dunlosky and Ariel (in press), however, Arabic readers did not favor easier items consistently across the arrays; that is, they did not consistently use an RPL agenda. One major difference between the methods of Experiment 1a and Dunlosky and Ariel (in press) was that the latter study involved the use of only two arrays: EMD or DME. Thus, perhaps native English readers would select items in a qualitatively different manner if they had to select items from a varying set of difficulty orders across trials (e. g., EMD, EDM, etc.), as we had used in Experiment 1a. In Experiment $1 \mathrm{~b}$, we evaluated this possibility by conducting Experiment 1a (with all six orders of item difficulty) with a group of native English readers.

\section{Method}

The procedure here was identical to that of Experiment 1a, with the following two exceptions: The task was presented in English to 24 native English-speaking undergraduate students from Kent State University, and the items were English-Spanish translations (18 easy, 18 moderately difficult, and 18 difficult) adapted from Metcalfe (2002).

Results and discussion

Means across participants' proportions of their first choice are presented in the right panel of Fig. 1 (values for subsequent choices conform to a reading bias, and hence are not reported). Data from Experiments $1 \mathrm{a}$ and $1 \mathrm{~b}$ are presented together in Fig. 1 to assist in comparing the qualitative patterns of selection between Arabic and English participants. However, we do not include an inferential comparison, because items differed across experiments. 
Fig. 1 Proportions of items chosen first for study in the left, middle, and right positions of the array in Experiments 1a (left panel) and $1 \mathrm{~b}$ (right panel). The presentation order conditions are presented on the $x$-axis: $\mathrm{E}=$ easy, $\mathrm{M}=$ moderately difficult, $\mathrm{D}=$ difficult. Error bars represent standard errors of the means

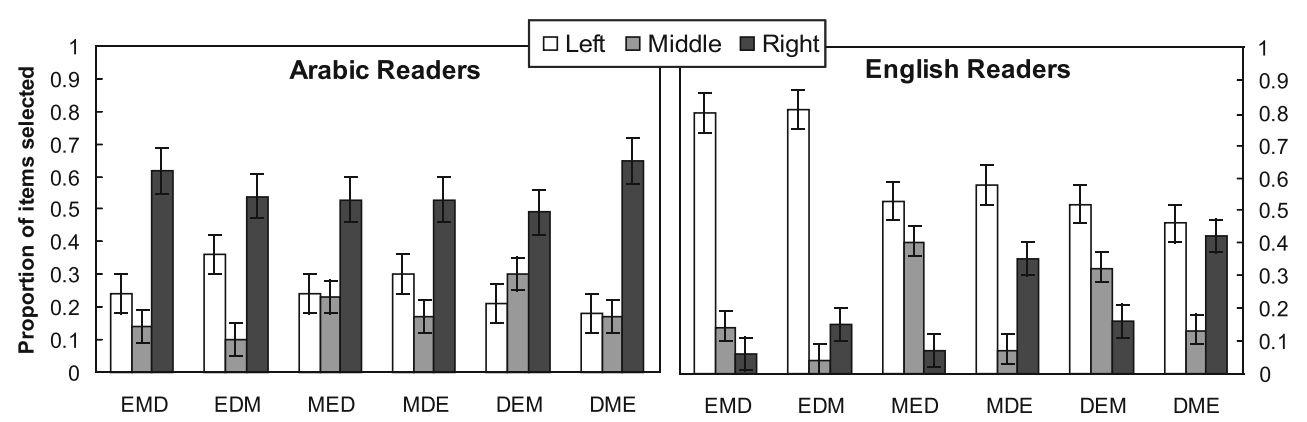

Contrary to the Arabic readers, English readers preferred to select items from the left position of the array more than items in other positions with their first choice. A 2 (position selected: left or right) $\times 6$ (presentation order) ANOVA yielded significant effects for position selected, $F(1,136)=$ $73.01, M S E=12.18, \eta_{\mathrm{p}}{ }^{2}=.35$, and presentation order, $F(5$, $136)=7.52, M S E=0.73, \eta_{\mathrm{p}}{ }^{2}=.22$, but these effects were qualified by a Position Selected $\times$ Presentation Order interaction, $F(5,136)=5.03, M S E=0.84, \eta_{\mathrm{p}}{ }^{2}=.16$. This interaction arose because preference for selecting items at the left rather than the right position of the array was magnified when easy items were presented at the left position (EMD and EDM conditions), $t \mathrm{~s}>6.57$, and diminished when easy items were presented at the right position (MDE and DME conditions), $t \mathrm{~s}<1.56$. Thus, English readers' decisions were influenced both by reading habits and by item difficulty, in a manner that reflected the use of an RPL agenda.

\section{Experiment 2}

Experiment $1 \mathrm{a}$ and $1 \mathrm{~b}$ used a cross-cultural analysis to examine whether directional reading habits bias study choices. In Experiment 2, we further explored the potential influence of reading habits on participants' study decisions by rotating lines of text $180^{\circ}$, so that each line of text was presented upside down. For example, the word easy would be presented as $\mathbf{\Lambda}$ sez. We expected that this manipulation would encourage some native English readers to select items from right to left, the rationale being that the beginning of each word was oriented rightward in space, and hence some participants might begin reading from right to left in this context. Of course, this attempt to invoke a right-to-left reading bias was unlikely to entirely override years of reading experience, so we did not expect it to completely reverse the order of item selection.

\section{Method}

Participants A group of 87 native English speaking students from Kent State University participated for course credit. A 6 (presentation order) $\times 2$ (text format: right-side up or upside down) mixed factorial design was used.
Participants were randomly assigned to either the rightside-up $(N=44)$ or the upside-down $(N=43)$ text group.

Materials and procedure The materials and procedure for Experiment 2 were identical to those of Experiment $1 \mathrm{~b}$ for the right-side-up group. The task was nearly identical for the upside-down group, with the major difference being that text was presented upside down by rotating each line of text $180^{\circ}$. The first four lines of text for the instructions were presented normally (right-side up). The upside-down group was instructed that our goal was to examine whether people could learn while reading text formatted upside down. They were told to read the instructions carefully and to start at the top-right corner of the screen. The task instructions were then presented rotated upside down. These instructions were the same as those presented to the right-side-up group but were presented upside down to get participants accustomed to reading from right to left. After participants finished the instructions, the experimenter confirmed that they understood the task.

The procedure for the study phase was exactly the same for the two text format groups, with the exception that the English words were presented upside down for one of the groups, in which case the difficulty order (easy, moderate, or difficult) was reversed relative to the normal English reading direction. Spanish translations were always presented right-side up, because they were difficult to read and because participants would type them right-side up during recall (in which all text was presented right-side up).

\section{Results and discussion}

The proportions of times participants selected items first for study are presented in Fig. 2. A 2 (text format) $\times 2$ (position selected) $\times 6$ (presentation order) ANOVA revealed significant effects for text format, $F(1,509)=$ 4.91, $M S E=0.20, \eta_{\mathrm{p}}{ }^{2}=.01$, position selected, $F(1,509)=$ $121.14, M S E=23.65, \eta_{\mathrm{p}}{ }^{2}=.19$, and presentation order, $F$ $(5,509)=20.80, M S E=0.83, \eta_{\mathrm{p}}{ }^{2}=.17$. These main effects were qualified by a Position Selected x Format interaction, $F(1,509)=63.51, M S E=12.40, \eta_{\mathrm{p}}{ }^{2}=.11$, and a Position Selected $\mathrm{x}$ Presentation Order interaction, $F$ 
Fig. 2 Proportions of items chosen first for study in the left, middle, and right positions of the array when text was presented right-side up (left panel) and upside down (right panel) in Experiment 2. Presentation order conditions are presented on the $x$-axis: $\mathrm{E}=$ easy, $\mathrm{M}=$ moderately difficult, $\mathrm{D}=$ difficult. Error bars represent standard errors of the means

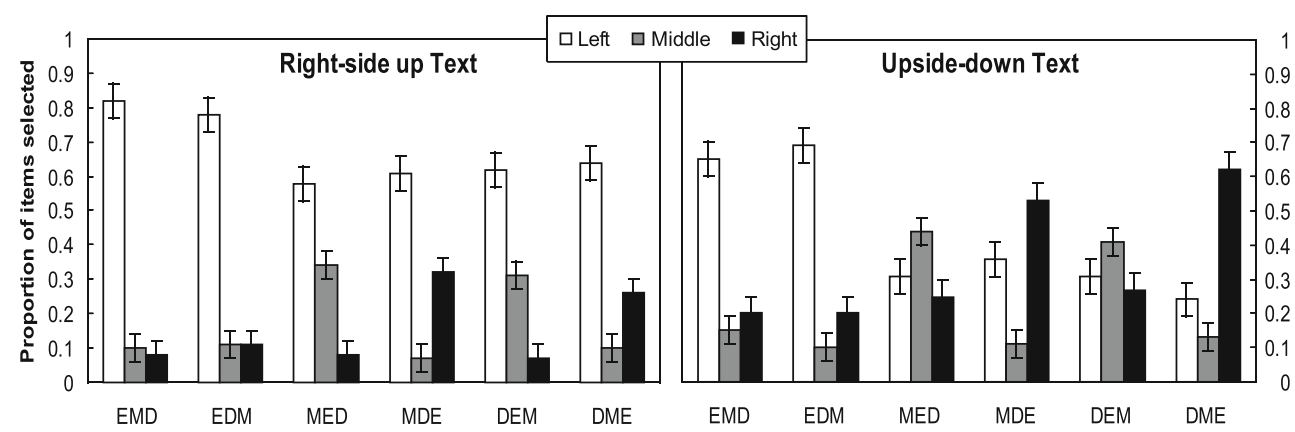

$(5,509)=14.13, M S E=2.76, \eta_{\mathrm{p}}{ }^{2}=.12$. The three-way interaction approached significance, $F(5,509)=2.15$, $M S E=0.42, p=.06, \eta_{\mathrm{p}}{ }^{2}=.02$.

To unpack these interactions, we conducted several followup tests that were most relevant to uncovering possible reading biases. In particular, critical comparisons involved examining preference as a function of position between groups; for instance, for the rightmost set of bars in each panel (DME conditions), the easy item (rightmost bar in each panel) is higher for the upside-down text format than for the right-sideup text format, indicating a greater propensity to choose from right to left when the text was upside down. Consistent with a reading-bias hypothesis, participants in the right-side-up group were more likely to first select items at the left position of the array (white bars in Fig. 2, collapsed across presentation orders: $M=.67, S E=.04$ ) than were participants in the upside-down group (collapsed across presentation orders: $M=$ $.44, S E=.04), t \mathrm{~s}>2.28$. In contrast, the upside-down group had a higher preference for first selecting items at the right position of the array (gray bars in Fig. 2, collapsed across presentation orders: $M=.33, S E=.04$ ) than did the right-sideup group (collapsed across presentation orders: $M=.15, S E=$ $.02), t \mathrm{~s}>2.05$. These patterns were consistent across all presentation orders, with the exception of the EDM condition, in which groups did not differ.

Inspection of Fig. 2 reveals that item difficulty influenced study decisions, in that participants often selected easy items first for study (heightened bars appear above "E" in most conditions). Even so, preference for selecting easy items only differed between groups in the EMD, MDE, and DME conditions, $t \mathrm{~s}>2.28$. That is, when easy items were in the left position (EMD), participants preferred to select them more in the right-side-up than in the upside-down group, and when they were presented in the right position (MDE and DME), participants preferred to select them more in the upside-down group. Thus, when easy items were likely to be read first, participants had a higher preference for selecting them first for study. In summary, upside-down text did not completely reverse participants' tendency to select items from left to right, but the influence of rotating text on item selection does provide converging evidence that reading habits bias study decisions.

\section{General discussion}

People's allocation of study time is influenced by multiple factors. Learners shape their study by constructing agendas aimed at achieving their goals, and yet habitual responding can capture their decision making and undermine agenda use. In the present experiments, participants' decisions were biased by the direction in which they read text in their native language, and as a consequence, many learners failed to allocate their study to items in the RPL. As with previous research involving cross-cultural analysis, our preferred interpretation is that these findings reflect the effects of a habitual reading bias on item selection.

In the present experiments, the effects of reading habits on study decisions were pitted against an RPL agenda. Some learners may have failed to realize that developing an RPL agenda would be an effective learning strategy, and in fact, no gains in performance were evident for selecting easy items first for study, as opposed to selecting other items first (see Table 1). This possibility provokes the question, Would reading habits undermine the use of agenda-based regulation in contexts where the agenda is more obvious and likely to impact performance? We began examining this question using a variation of the design from the present experiments in which item order was placed in opposition to the reward associated with learning items (Ariel \& Dunlosky, 2010). On each trial, participants were presented with a lowreward (1 point), a medium-reward (3 points), and a highreward (5 points) item, and participants had $5 \mathrm{~s}$ to select and study the items. An obvious strategy to maximize performance (in terms of points earned) would be to prioritize the 5-point items for study, and previous research indicated that learners typically develop agendas to study highly valued items (Ariel et al., 2009; Castel, 2007; Castel, Farb, \& Craik, 2007). Accordingly, we expected that the influence of reading habits would weaken in this context, but participants' study decisions were still largely biased by reading habits, even when participants had a monetary incentive to earn as many points as possible! 
Another explanation why learners did not consistently use an RPL agenda in the present experiments could be that they had enough time to choose all three items for study. Although this explanation was possible, it is not consistent with results from the following pilot experiment: Arabic readers selected items for study from an EMD array either under a 5-s time pressure or under no time pressure (unlimited time). They were just as likely to select items in the right position of the array (in this case, the difficult item) when studying under time pressure $(M=.56, S E=$ $.06)$ as when under no time pressure $(M=.59, S E=.06)$. Thus, in the present context, even when Arabic readers could not select all items for study, their selection decisions were still biased by reading habits.

We suspect that in many contexts learners do not develop agendas to regulate their study effectively, but instead rely on habitual responding to regulate their study time. For example, students studying for exams may study items in the order in which they appear in textbooks and notes instead of using an agenda that would help the students more efficiently obtain the course-related goals, such as by allocating time first to the most highly valued items (e.g., those that are more likely to be on the exam) or to their RPL. Based on this possibility, understanding what triggers agenda development is an important goal for future research. What is evident from the present results, however, is that learners' study decisions can be influenced by a habitual reading bias that can undermine the use of other agendas.

Author note This research was supported by a Collaborative Award from the James S. McDonnell Foundation 21st Century Science Initiative in Bridging Brain, Mind and Behavior.

\section{Appendix}

Table 1 Proportions of items recalled as a function of item difficulty in each experiment

Values are means across individual participants' proportions of correct recall. Standard errors of the means are in parentheses. Only effects for item difficulty were significant, $F$ s $>28.28$, with recall for easier items $>$ recall for moderately difficult ones $>$ recall for difficult ones.

\begin{tabular}{lllllll}
\hline & EMD & EDM & MED & MDE & DEM & DME \\
\hline Experiment 1a & & & & & & \\
Easy & $.32(.06)$ & $.35(.06)$ & $.35(.06)$ & $.32(.06)$ & $.35(.06)$ & $.31(.06)$ \\
Moderate & $.10(.04)$ & $.10(.03)$ & $.14(.04)$ & $.07(.04)$ & $.14(.04)$ & $.17(.06)$ \\
Difficult & $.00(.00)$ & $.00(.00)$ & $.01(.01)$ & $.01(.01)$ & $.01(.01)$ & $.01(.01)$ \\
Experiment $1 \mathrm{~b}$ & & & & & & \\
Easy & $.49(.06)$ & $.56(.07)$ & $.47(.07)$ & $.40(.07)$ & $.44(.07)$ & $.51(.07)$ \\
Moderate & $.17(.05)$ & $.19(.06)$ & $.15(.05)$ & $.04(.02)$ & $.19(.06)$ & $.15(.06)$ \\
Difficult & $.04(.04)$ & $.00(.00)$ & $.00(.00)$ & $.01(.01)$ & $.00(.00)$ & $.01(.01)$ \\
Experiment 2 & & & & & & \\
Right-side-up text & & & & & & \\
Easy & $.58(.05)$ & $.52(.05)$ & $.47(.05)$ & $.52(.05)$ & $.51(.05)$ & $.50(.05)$ \\
Moderate & $.12(.04)$ & $.20(.05)$ & $.05(.01)$ & $.10(.03)$ & $.10(.03)$ & $.09(.02)$ \\
Difficult & $.04(.02)$ & $.01(.01)$ & $.02(.01)$ & $.00(.00)$ & $.02(.01)$ & $.01(.01)$ \\
Upside-down text & & & & & & \\
Easy & $.55(.05)$ & $.56(.06)$ & $.48(.06)$ & $.46(.05)$ & $.46(.05)$ & $.49(.06)$ \\
Moderate & $.09(.04)$ & $.10(.00)$ & $.09(.04)$ & $.15(.04)$ & $.06(.03)$ & $.14(.04)$ \\
Difficult & $.04(.03)$ & $.04(.02)$ & $.06(.04)$ & $.06(.03)$ & $.02(.02)$ & $.05(.03)$ \\
\hline
\end{tabular}

\section{References}

Ariel, R., \& Dunlosky, J. (2010). The influence of rewards for performance and habitual responding on agenda-based regulation of study. Poster presented at the 51st Annual Meeting of the Psychonomic Society, St. Louis, MO.

Ariel, R., Dunlosky, J., \& Bailey, H. (2009). Agenda-based regulation of study-time allocation: When agendas override item-based monitoring. Journal of Experimental Psychology. General, 138, 432-447. doi:10.1037/a0015928
Atkinson, R. C. (1972). Optimizing the learning of a second-language vocabulary. Journal of Experimental Psychology, 96, 124-129. doi: $10.1037 / \mathrm{h} 0033475$

Castel, A. D. (2007). The adaptive and strategic use of memory by older adults: Evaluative processing and value-directed remembering. In A. S. Benjamin \& B. H. Ross (Eds.), The psychology of learning and motivation: Skill and strategy in memory use (Vol. 48, pp. 225-270). Orlando: Academic.

Castel, A. D., Farb, N. A. S., \& Craik, F. I. M. (2007). Memory for general and specific value information in younger and older adults: Measuring the limits of strategic control. Memory \& Cognition, 35, 689-700. 
Chokron, S., \& De Agostini, M. (1995). Reading habits and line bisection: A developmental approach. Cognitive Brain Research, 3, 51-58. doi:10.1016/0926-6410(95) 00018-6

Chokron, S., \& De Agostini, M. (2000). Reading habits influence aesthetic preference. Cognitive Brain Research, 10, 45-49. doi:10.1016/S0926-6410(00)00021-5

Dunlosky, J., \& Ariel, R. (2011). Self-regulated learning and the allocation of study time. In B. H. Ross (Ed.), The psychology of learning and motivation: Advances in research and theory (Vol. 54, pp. 103-140). San Diego: Academic.

Dunlosky, J., \& Ariel, R. (in press). The influence of agenda-based and habitual processes on item selection during study. Journal of Experimental Psychology: Learning, Memory, and Cognition. doi:10.1037/a0023064

Eviator, Z. (1995). Reading direction and attention: Effects on lateralized ignoring. Brain and Cognition, 29, 137-150.

Kornell, N., \& Metcalfe, J. (2006). Study efficacy and the region of proximal learning framework. Journal of Experimental Psychology. Learning, Memory, and Cognition, 32, 609-622. doi:10.1037/0278-7393.32.3.609
Metcalfe, J. (2002). Is study time allocated selectively to a region of proximal learning? Journal of Experimental Psychology. General, 131, 349-363. doi:10.1037/0096-3445.131.3.349

Metcalfe, J. (2009). Metacognitive judgments and control of study. Current Directions in Psychological Science, 18, 159-163. doi:10.1111/j.1467-8721.2009.01628.x

Ouellet, M., Santiago, J., Israeli, Z., \& Gabay, S. (2010). Is the future the right time? Experimental Psychology, 57, 308-314. doi:10.1027/1618-3169/a000036

Shaki, S., Fischer, M. H., \& Petrusic, W. M. (2009). Reading habits for both words and numbers contribute to the SNARC effect. Psychonomic Bulletin \& Review, 16, 328-331. doi:10.3758/PBR.16.2.328

Son, L. K., \& Kornell, N. (2009). Research on the allocation of study time: Key studies from 1890 to the present (and beyond). In J. Dunlosky \& R. A. Bjork (Eds.), A handbook of metamemory and memory (pp. 333-351). New York: Psychology Press.

Spalek, T. M., \& Hammad, S. (2005). The left-to-right bias in inhibition of return is due to the direction of reading. Psychological Science, 16, 15-18.

Van der Henst, J.-B., \& Schaeken, W. (2005). The wording of conclusions in relational reasoning. Cognition, 97, 1-22. doi:10.1016/j.cognition.2004.06.008 